\title{
Millimeter wave performance of a sliding planar backshort
}

\section{Victor M. Lubecke}

Victor M. Lubecke, "Millimeter wave performance of a sliding planar backshort," Proc. SPIE 2250, International Conference on Millimeter and Submillimeter Waves and Applications 1994, 22507W (30 November 2017); doi: $10.1117 / 12.2303301$

SPIE Event: Millimeter and Submillimeter Waves and Applications: International Conference, 1994, San Diego, CA, United States 


\title{
Millimeter wave performance of a sliding planar backshort
}

\author{
Victor M. Lubecke, ${ }^{1}$ William R. McGrath, ${ }^{2}$ and David B. Rutledge ${ }^{1}$ \\ 1. Department of Electrical Engineering, \\ California Institute of Technology, Pasadena, CA, 91125 \\ 2. Center for Space Microelectronics Technology, Jet Propulsion Laboratory, \\ California Institute of Technology, Pasadena, CA, 91109
}

\begin{abstract}
A mechanically adjustable planar tuning element, for millimeter and submillimeter wave planar integrated circuits, has been developed and successfully demonstrated at $100 \mathrm{GHz}$. It functions analogously to a non-contacting waveguide backshort, with $\left|s_{11}\right| \simeq-0.3 \mathrm{~dB}$, yet can be fabricated with the simplicity of a planar circuit and scaled for use throughout the millimeter and submillimeter wave spectrum.
\end{abstract}

\section{INTRODUCTION}

Parasitic reactance in various devices can seriously degrade their performance in millimeter wave and submillimeter wave applications. While effective, waveguide embedding circuits which use mechanically adjustable backshorts to tune out the undesired reactance become more costly and difficult to fabricate as the design frequency increases. Planar circuits offer an attractive alternative, but typically do not offer a means of post-fabrication optimization. Presented here is a frequency scalable planar circuit element which can be mechanically adjusted to optimize the performance of a planar circuit, functioning analogously to an insertable backshort in a waveguide circuit. Realizations for both millimeter wave $(100 \mathrm{GHz})$ and submillimeter wave $(620 \mathrm{GHz})$ applications of this design are presented and the RF performance of the milimeter wave application is examined.

\section{Design, Fabrication, and Test}

The sliding planar backshort discussed here consists of a movable metal sheet or plate, with appropriately sized and spaced holes, placed on top of a dielectric-coated planar transmission line. In this position, the metal pattern creates a sequence of alternating low and high impedance quarter-wavelength sections of transmission line, resulting in a non-contacting, broadband RF short circuit. Critical dimensions for the sliding plate and transmission line are scaled, by frequency, from an empirically designed $2 \mathrm{GHz}$ model [1].

The $100 \mathrm{GHz}$ sliding short, shown in Fig. 1, was fabricated as an evaporated $\mathrm{Cr}$-Ag-Cr pattern supported by a small, movable quartz chip. It was placed, metal-side-down, on top of a planar test circuit consisting of a $\mathrm{Cr}$-Au coplanarstrip transmission line and planar dipole antenna, evaporated onto a quartz substrate and covered by a submicron

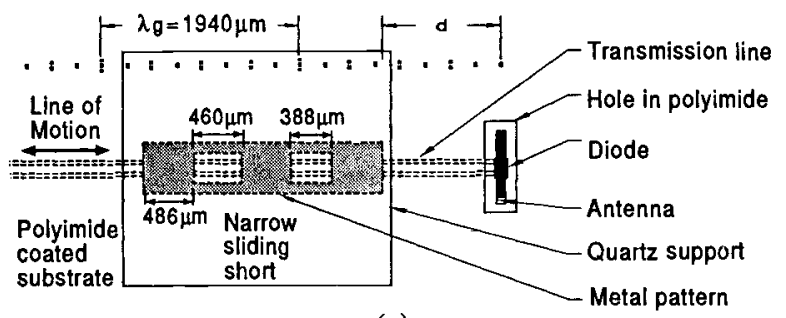

(a)

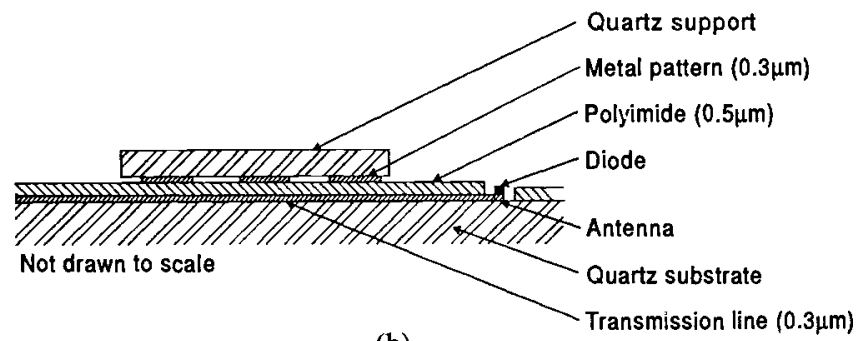

(b)

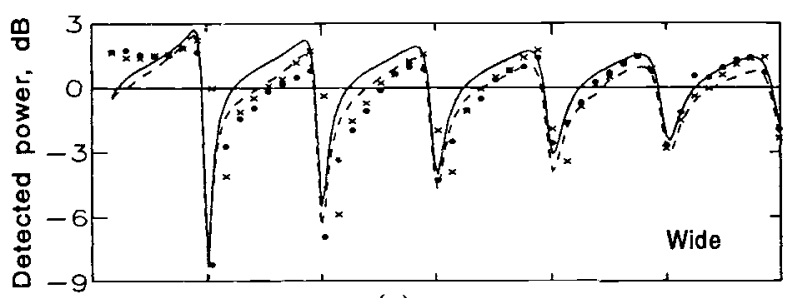

(a)

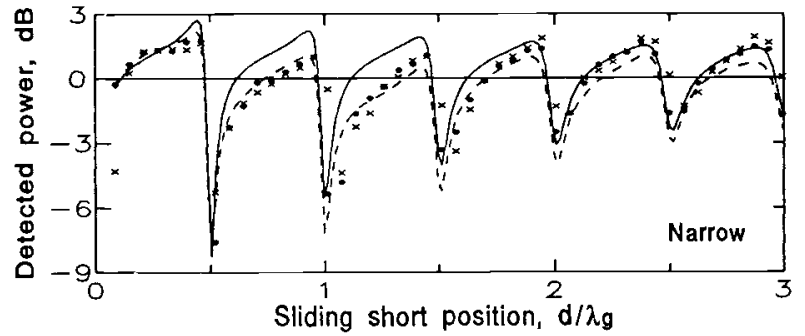

(b)
Fig. 1. Top view (a) and cross section (b) of the mechanically tunable planar detector circuit.

International Conference on Millimeter and Submillimeter Wavect, shown for both wide (a) and narrow (b) sliding shorts. Proc of SPIE Vol. 2250, 22507W · (1994) 2017 SPIE .
Fig. 2. Two trials $(\bullet)(x)$, without $(-)$, and with $(--)$ coupling effect, shown for both wide (a) and narrow (b) sliding shorts. CCC code: $0277-786 X / 17 / \$ 18 \cdot$ doi: $10.1117 / 12.2303301$ 
layer of polyimide. A GaAs Schottky beam-lead diode, used as a detector, was soldered across the antenna. A more detailed description of the fabrication and test of this circuit can be found in a separate publication [2].

Measurements were made with the detector circuit illuminated by a modulated $100 \mathrm{GHz}$ source. The detected power, measured with a lock-in amplifier, was recorded with the sliding short manually positioned at various distances, $d$ in Fig. 1, along the transmission line. Measurements were repeated using two sliding short designs, both having identical dimensions along the transmission line, but one with a wider metallization pattern across the line. Detected power, normalized to the response with no backshort present $(\simeq 160 \mu \mathrm{V})$, is shown in Fig. 2 as a function of the sliding short position, normalized to the guide wavelength for the coplanar strip transmission line $\left(\lambda_{g}\right)$.

A theoretical model was developed for this circuit and is also shown in Fig. 2. The model includes calculations for both conductor and radiation losses for the 190- $\Omega$ coplanar strip transmission line and uses $\left|s_{11}\right|=-0.3 \mathrm{~dB}$ for the sliding short, as measured in the $2 \mathrm{GHz}$ experiment. A parasitic coupling effect between the sliding short and antenna was also observed. An attempt was made to measure this coupling and the effect was added to the theoretical response; this is also shown in Fig. 2.

A $620 \mathrm{GHz}$ version of the sliding short was also fabricated. The captivated sliding element was, in this case, formed by a combination of techniques borrowed from silicon surface micromachining and LIGA [3], illustrated in Fig. 3. It involves depositing the gold sliding element, by electroplating with a mold, between two sacrificial layers of copper. An overhanging polyimide guiding structure is then added in order to captivate the sliding element once the copper is etched away. This process resulted in a movable metallic tuning plate, with one degree of freedom, which can be readily fabricated on top of a planar transmission line to form a fully integrated, micromechanical tuning element. This sliding short is shown in Fig. 4.

\section{ACKNOWLEDGEMENTS}

We would like to thank Dr. P.A. Stimson, Dr. P.H. Siegel, and Dr. Y-C. Tai for their invaluable assistance. The research described in this paper was jointly sponsored by the Innovative Science and Technology Office of the Ballistic Missile Defense Organization, and the NASA Office of Advanced Concepts and Technology. V.M. Lubecke holds a NASA GSRP Fellowship.

\section{REFERENCES}

[1] V.M. Lubecke, W.R. McGrath, and D.B. Rutledge, "Sliding Backshorts for Planar Circuits," International Journal of Infrared and Millimeter Waves, vol. 12, no. 12, pp. 1387-1397, December 1991.

[2] V.M. Lubecke, W.R. McGrath, and D.B. Rutledge, "A $100 \mathrm{GHz}$ Coplanar Strip Circuit Tuned with a Sliding Planar Backshort," submitted to IEEE Microwave and Guided Wave Letters, July 1993

[3] A. Bruno and M.G. Allen, "Metallic Microstructures Fabricated Using Photosensitive Polyimide Electroplating Molds," IEEE Journal of Micromechanical Systems, vol. 2, no. 2, pp. 87-94, June 1993.

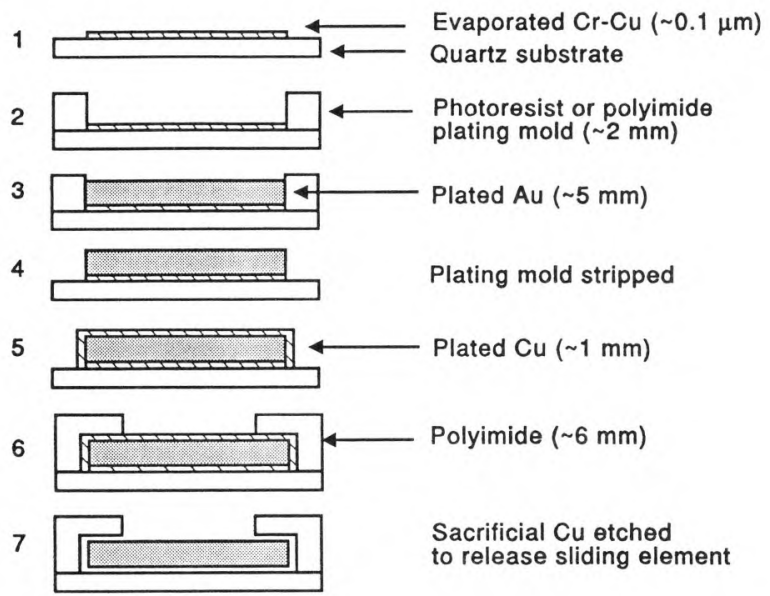

(a)

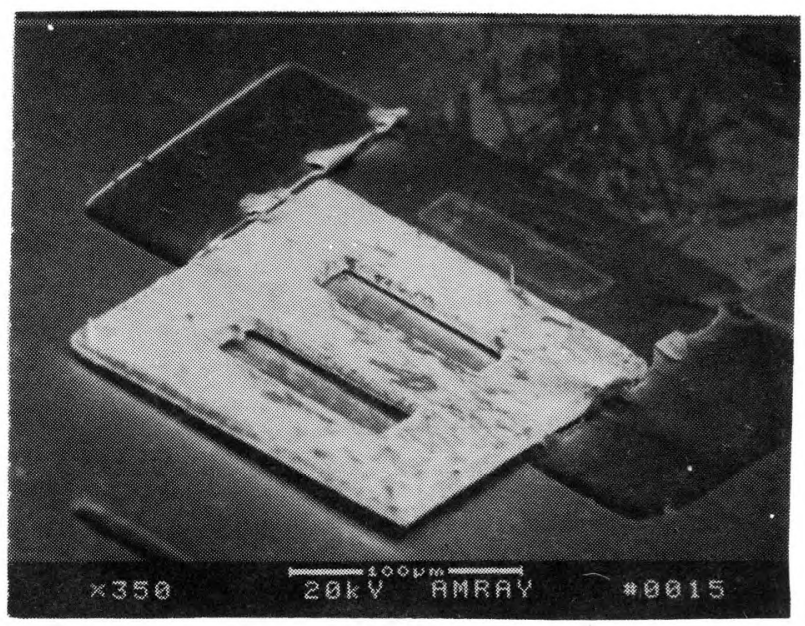

(b)

Fig. 3. Fabrication process (a) and SEM photograph (b) of $620 \mathrm{GHz}$ sliding element. 